\title{
JUECES Y «CONTROL DE CALIDAD» DE LOS SISTEMAS POLÍTICOS
}

\author{
María José Añón \\ Universidad de Valencia
}

RESUMEN. Este artículo trata de analizar la tesis normativa que atribuye a los jueces la función de garantía de la estabilidad de los sistemas políticos, examen que se lleva a cabo en dos planos. En primer lugar, realizo una valoración de la contribución de la regla de reconocimiento a la identidad y a la legitimidad de los sistemas jurídicos. Planteo, después, la idea de que el rasgo distintivo de la función judicial desde un punto de vista institucional y normativo no es tanto el mantenimiento de la estabilidad del orden político cuanto la garantía de los derechos, subrayando las limitaciones de la tesis analizada en este artículo en contextos de transformación del orden jurídico-político. dad.

Palabras clave: Ernesto Garzón Valdés, función judicial, estabilidad política, legitimi-

ABSTRACT. This article intends to analyse the normative thesis that assigns the function of safeguarding the stability of political systems to judges. The exam is made in two levels. First, I evaluate how the rule of recognition contributes to strengthen both the identity and the legitimacy of legal systems. By pointing out the limits of the stability thesis in contexts of legal and political transformation, I pose afterwards that the outstanding institutional and normative feature of judicial function is not the sustenance or the reinforcement of political stability, but the protection of rights.

Keywords: Ernesto Garzón Valdés, judicial function, political stability, legitimacy. 


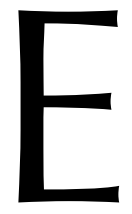

n su trabajo «El papel del poder judicial en la transición a la democracia» ${ }^{1}$, Ernesto GARZÓN reflexiona proyectivamente sobre las condiciones de posibilidad de los jueces en los procesos de transición hacia la democracia. El texto del artículo es agudo, incisivo y cargado de sugerencias imposibles de atender en profundidad en esta sede. Sucintamente cabe afirmar que el autor asume los rasgos caracteriológicos del juez del Estado constitucional y cifra en tres puntos básicos las exigencias que deberían ser asumidas por los jueces de las instancias más elevadas: la limitación estricta del control de calidad democrática a los principios básicos del sistema; el mantenimiento de una rigurosa independencia del poder judicial y la introducción de un paradigma material en la interpretación de los principios constitucionales con objeto de que la sociedad alcance un grado de homogeneidad socio-económica que permita la homogeneidad de derechos².

El artículo sostiene la tesis de que los jueces de tribunales superiores en contextos democráticos son una suerte de «inspector de calidad», de acuerdo con la expresión de L. SAGER ${ }^{3}$, del sistema democrático y de sus instituciones y poderes. Esta idea se sustenta y proviene de una afirmación normativa sobre el poder judicial que considera que «su función principal es la de garantizar la estabilidad del respectivo sistema político» ${ }^{4}$. Afirmación que - continúa E. GARZÓN-vale para todo sistema político.

Quiero limitar mi intervención a examinar esta tesis, introduciendo algunas matizaciones al desarrollo argumentativo de la misma. Para ello, resulta preciso comprender las bases sobre las que se sustenta la anunciada proposición. La estabilidad es, tal como la define GARZÓN ${ }^{5}$, una propiedad disposicional de un sistema político a reaccionar de forma tal que éste mantenga su identidad, nutriéndose de la actitud de quienes detentan el poder de orientar su comportamiento de acuerdo con las normas básicas del sistema. Esta posición se asienta, a su vez, en dos exigencias normativas. Los jueces, afirma GARZÓN, deben cultivar «una firme adhesión interna a las normas básicas del sistema», dado que la connotación moral de este punto de vista es expresión de la autonomía del poder judicial y, en segundo término, deben mantener «una manifiesta imparcialidad respecto a los conflictos que deben resolver» ${ }^{6}$.

En este sentido, GARZÓN distingue certeramente entre estabilidad de un sistema y legitimidad. El juicio de legitimidad es de naturaleza normativa y su justificación es, en última instancia, de tipo moral, puesto que hablamos de la calidad ética de un sistema político. Por su parte, el juicio de estabilidad es descriptivo, y su verdad o falsedad depende de la existencia de características empíricamente comprobables, sin que importe la calidad moral de las acciones que las provocaron ${ }^{7}$. Puede afirmarse así que un sistema político es estable cuando, en determinadas circunstancias, tiene la tendencia o

\footnotetext{
${ }^{1}$ Artículo publicado en Isonomía, n. ${ }^{\circ}$ 18, abril 2003, pp. 27-46. También forma parte del libro MALEM; OROZCO y VÁZQUEZ (comps.), 2003: Barcelona: Gedisa, pp. 129-147.

2 GARZÓN, 2003: 43.

3 SAGER, 1998: «The Domain of Constitutional Justice», en AlEXANDER (ed.), 1998: Constitutionalism. Philosophical Foundations, Cambridge University Press: p. 238. Citado por GARZÓN, $2003: 30$.

${ }^{4}$ GARZÓN, 2003: 27.

5 GARZÓN, 1987: «El concepto de estabilidad de los sistemas políticos», Derecho, Ética y Política, Introducción de M. ATIEnZA, Madrid: Centro de Estudios Constitucionales, pp. 573-609.

${ }^{6}$ GARZÓN, 2003: 27-28.

7 GARZÓN, 2003: 573, 595.
} 
disposición a reaccionar en forma tal que logra mantener su identidad, esto es, sus cambios son una explicación de su «regla de reconocimiento»

La regla de reconocimiento es caracterizada por el autor como la base de la identidad y unidad del sistema jurídico-político, en tanto que indica los criterios de validez y membrecía de las reglas de un sistema. La existencia de un sistema político-jurídico está estrechamente vinculada con el punto de vista interno: un sistema existe sólo si al menos el grupo dominante acepta la regla de reconocimiento desde el punto de vista interno y es capaz de imponer su regla de reconocimiento en la respectiva sociedad, lo que se pone de manifiesto a través de la obediencia y el uso de las reglas válidas del mismo; siendo la magnitud del grupo dependiente de los diferentes tipos de organización política.

La regla de reconocimiento — escribe HART— «existe como una práctica compleja, pero normalmente concordante, de los tribunales, funcionarios y particulares, al identificar el Derecho por referencia a ciertos criterios» ${ }^{8}$. Lo relevante, a estos efectos, es su consideración, desde el punto de vista interno, como criterio común y público de decisiones judiciales correctas, y no como algo que cada juez, simplemente obedece por su cuenta $^{9}$. RUIZ MANERO afirma, al respecto, que la mera existencia de una práctica concordante de identificación de reglas en relación a ciertos criterios por parte de jueces y tribunales no es condición suficiente para la existencia de la regla de reconocimiento, sino que tales criterios de validez jurídica han de ser considerados por los órganos de aplicación en general, como un criterio común de decisiones judiciales correctas, valorando críticamente las desviaciones respecto a los criterios vigentes. Según HART, «la afirmación de que un sistema jurídico existe es, por lo tanto, un enunciado bifronte, una de cuyas caras mira a la obediencia por parte de los ciudadanos ordinarios y la otra a la aceptación de reglas secundarias como pautas o criterios comunes críticos de conducta oficial, por parte de los funcionarios» ${ }^{10}$. Por tanto, la adopción del punto de vista interno, la creencia de que la regla de reconocimiento es correcta o la aprobación de la misma hacen referencia a una misma realidad: la aceptación de las reglas del sistema es un elemento esencial de la definición de legitimación. No siguiéndose de esta afirmación —escribe E. GARZÓN ${ }^{11}$ — ninguna proyección relativa a su estabilidad y tampoco sobre su legitimidad.

Calibrar esta idea exige distinguir dos planos a propósito de la regla de reconocimiento, el que da cuenta de su práctica efectiva y, por tanto, de la identificación de un sistema jurídico, desde un punto de vista externo y la pregunta por la justificación de la misma. Esto plantea la cuestión del carácter que tienen los enunciados que afirman no que la regla de reconocimiento existe, sino que los jueces deben hacer lo que ella prescribe, es decir, que deben fundamentar sus decisiones en las reglas identificadas a partir de los criterios de validez contenidos en dicha regla. En este caso, como escribe J. RUIZ MANERO ${ }^{12}$, cuando se afirma que la regla de reconocimiento de un determina-

${ }^{8}$ HarT, 1968: El concepto de Derecho, Buenos Aires: Abeledo-Perrot, traducción de G. R. CARRIÓ, p. 137.

9 HART, 1968: 128, 144-145.

${ }^{10}$ HART, 1968: 145.

${ }^{11}$ GARZÓN, 1993: 585.

12 Ruiz MANero, 1990: Jurisdicción y normas, CEC: 121, 173. Sobre las implicaciones de la distinción entre razones explicativas y justificativas en relación a la aceptación por parte de los aplicadores del Derecho, véase BAYÓn, La normatividad del Derecho, Epílogo, pp. 731 y ss. y HIERRO, 2002: «¿Por qué ser positivista?», Doxa, n. ${ }^{\circ}$ 25, p. 264. 
do sistema jurídico exige de los jueces que apliquen los estándares que reúnan los requisitos establecidos por la propia norma, quiere decirse que la regla de reconocimiento prescribe a los jueces que consideren tales estándares como razones jurídicas independientes del contenido o autoritativas. Aceptar la regla de reconocimiento viene a consistir así en aceptar desde una actitud crítica reflexiva, considerar los estándares jurídicos como razones perentorias para la acción.

Desde el punto de vista explicativo de la motivación de los jueces para tal aceptación, puede exhibirse un amplio abanico de razones de distinto orden. Sin embargo, desde el punto de vista justificatorio o conceptual, no cabe suscribir cualquier tipo de razón, como argumenta RUIZ MANERO. La aceptación de la regla de reconocimiento no podría justificarse mediante razones jurídicas, dado su carácter de norma última, ni prudenciales ni en razones de autointerés, sino en razones morales, puesto que se trata de una regla que impone deberes sobre otros. Aceptar la regla de reconocimiento por razones morales no equivale, como cree HART, a asumirla por una creencia en la legitimidad moral de las fuentes señaladas por la regla de reconocimiento ${ }^{13}$. La aceptación por razones morales por parte de los jueces, porque éstos consideran que tienen la obligación moral de seguir las normas como razones perentorias, supone que la regla de reconocimiento no es una condición de estabilidad, sino de existencia, como reconoce GARZÓN VALDÉS. Seguramente, la posición de los jueces tiene más que ver con la legitimidad del sistema que con su estabilidad, dado que la regla de reconocimiento es un criterio de existencia, desde el punto de vista externo y un criterio de justificación, desde el punto de vista interno, al menos para los jueces.

Ahora bien — retomaremos esta idea posteriormente— la regla de reconocimiento del sistema sólo se puede identificar, como advierte HART ${ }^{14}$, «en condiciones normales de armonía oficial y, especialmente de la armonía judicial». Sin embargo, cuando nos encontramos ante el supuesto de un sistema jurídico embrionario o in statu nascendi, es decir, en una fase de transición, la dificultad básica reside en determinar cuál es exactamente la regla de reconocimiento o los cambios que afectan a la propia regla de reconocimiento. Esto es lo característico de las transformaciones o cambios jurídico-políticos de orden, digamos, fundacional, que dan carta de naturaleza a un nuevo sistema, como ocurre precisamente, no cuando se modifican a las normas del sistema jurídico y podemos seguir hablando de permanencia del mismo sistema, sino cuando los cambios afectan a las normas últimas o independientes.

Llegamos a la segunda de las cuestiones que quisiera abordar. Esto es, una aproximación a algunas consecuencias de las garantías institucionales del juez en la transformación del orden jurídico-político. En este ámbito encuentra su sentido la pregunta por el papel de los jueces en la transición a un sistema jurídico-político democrático. La respuesta tentativa precisa llevar a cabo una valoración conjunta tanto de las condiciones de legitimidad formal, cuando de legitimidad material de la judicatura y de su responsabilidad como garante de los derechos.

Ciertamente, no es demasiado preciso lo que puede afirmarse sobre el juez en abstracto y las dificultades que de ahí se derivan, puesto que la mayor parte de sus propiedades institucionales responden a algún modelo de juez vinculado a una cultura jurídi-

\footnotetext{
13 Ruiz Manero, 1990: 197.

14 HART, 1968: 152.
} 
ca más o menos determinada. En términos generales es posible describir la jurisdicción como una función del Estado destinada a decidir con imparcialidad sobre situaciones controvertidas según las reglas del Derecho vigente. La nota que proporciona mayor identidad a los jueces es la figura de un tercero imparcial que interviene en la resolución de conflictos, cuyas decisiones se legitiman por una justificación aceptable de la verdad de los presupuestos fácticos y jurídicos.

Las condiciones del juez en abstracto no cuentan con una determinación exacta, pero entre ellas destacan las exigencias de legitimidad formal procesales o institucionales y un criterio de legitimidad sustancial ${ }^{15}$. La legitimidad material descansa en la vinculación del juez a la ley y se manifiesta en las funciones de garantía de los derechos y de solución de las controversias asignadas a la jurisdicción frente o contra los demás poderes, utilizando, entre otros instrumentos, su poder de censurar las propias leyes en cuanto inconstitucionales. Éste es el modelo jurisdiccional propio del Estado Constitucional, al que corresponden distintas tesis definitorias y justificativas derivadas de distintas versiones del neoconstitucionalismo ${ }^{16}$. Entre las garantías formales que surgen de las virtudes pasivas de los jueces a las que se refiere CAPPELLETTI, destacarían las siguientes ${ }^{17}$. Reglas procesales que exigen: i) que el juez actúe como un tercero suprapartes, que no decida en una controversia en la que él es participe, y que no esté sometido a presiones; ii) que el proceso tenga efectivamente carácter contradictorio, donde las partes tengan efectivamente posibilidades de defenderse, ser oídas; iii) que el proceso judicial tenga un carácter pasivo, que precisa la acción de un actor que inicia el proceso; iv) que el juez motive sus decisiones.

Entre estas garantías se encuentra la independencia. Ernesto GARZÓN parte de la autonomía judicial como condición necesaria para hablar de la existencia de un sistema jurídico. Esta autonomía proviene o deriva de la connotación moral del punto de vista interno propio de los jueces, que les distancia y separa del espacio político propio de la razón instrumental, articulado a través de la negociación y el compromiso ${ }^{18}$. No puedo detenerme en profundizar sobre el peso de lo político, dicho con toda la amplitud, tanto en la estabilidad como en la legitimidad de los sistemas jurídico-políticos y me limitaré a apuntar unas sucintas ideas sobre las notas definitorias de la autonomía judicial en términos de autonomía de la política.

Cuando se habla de autonomía o de independencia externa suele hacerse referencia a una posición institucional de la judicatura considerada como poder del Estado. Autonomía e independencia, como sugiere GARCía PASCUAL ${ }^{19}$, no son la misma cosa, pues mientras que la independencia es una garantía jurídico-institucional que se impone al juez en calidad de principio regulativo, el concepto de autonomía hace referencia a la institución o poder del Estado.

${ }_{15}$ García Pascual, Legitimidad democrática: 176-178. Prieto, 1987: Ideología e interpretación jurídica, Madrid: Tecnos.

${ }_{16}$ Para una interesante distinción del neoconstitucionalismo como ideología, como teoría y como método, véase COMANDUCCI, 2005: «Formas de (neo)constitucionalismo: un análisis metateórico», Neoconstitucionalismo(s), M. CARBONELL (ed.), Madrid: Trotta, pp. 75-98.

${ }^{17}$ CAPPELletTI, 1988: Giudici irresponsabili?, Milan: Giuffrè, p. 66.

${ }^{18}$ GARZÓn, 2003: 27. Sobre las posibilidades de establecer puentes entre el ámbito de la argumentación y el de la negociación, véase DE PÁRAMO y CALVO.

${ }^{19}$ García Pascual, Legitimidad democrática y poder judicial, cit, p. 163. 
En este sentido, considero que la cualidad que mejor define a los jueces, vinculada a las connotaciones del punto de vista interno, es más la independencia personal como ideal o idea regulativa que la autonomía. Siguiendo una interesante tesis de AGUILÓ ${ }^{20}$, los jueces no son independientes porque están sometidos sólo al Derecho, sino que tienen además un deber de ser independientes, evidentemente en un marco institucional idóneo para que ello sea posible. Este deber consiste en cumplir el Derecho, de ahí que se afirme que la independencia es la peculiar forma de cumplimiento que el Derecho exige a los jueces, que son autoridades vinculadas al orden jurídico - como todas las demás- y con un deber de independencia. Esta peculiar forma de cumplir el Derecho está vinculada a su legitimidad, producto de una combinación de legalidad e independencia. En este sentido «el juez no puede ser portador de fines o intereses extraños al Derecho» ${ }^{21}$. El deber de independencia del juez tiene como correlato el derecho de los ciudadanos a ser juzgados desde el Derecho y no desde parámetros extrajurídicos provenientes del sistema social. Independiente es el juez que aplica el Derecho (actúa en correspondencia con el deber) y que lo hace movido por las razones que el Derecho le suministra (actúa movido por el deber).

La ductilidad que exhiben los jueces ante los cambios y transformaciones de los sistemas político-jurídicos nos permiten comprender mejor el alcance de la afirmación hartiana de que la regla de reconocimiento sólo puede determinarse en períodos «armonía jurídica». Veamos dos ejemplos de distinto signo que pueden ilustrar esta idea. De un lado, la versatilidad del juez napoleónico y su capacidad de integración en distintas experiencias autoritarias ${ }^{22}$. Los diferentes fascismos europeos y dictaduras militare se asentaron, sin apenas reformas sustanciales en la legislación procesal y gracias a la (relativa) continuidad de la regulación de la organización (administrativa) de la judicatura como poder, aunque en este último aspecto las dictaduras se han preocupado bastante de asegurarse un poder judicial afecto, que atribuyó a los jueces una consideración central como factor de control social antes que autoridades garantes de los derechos. De ahí, como subraya ANDRÉS IBÁÑEZ ${ }^{23}$, que fueran profundamente cuestionados en los albores del constitucionalismo emergente tras la segunda guerra mundial y que la experiencia dramática de los fascismos europeos llevara, no sólo a predisponer fuertes límites de derecho al ejercicio de la política, al objeto de afianzar el sometimiento del juez a la ley, sino también a establecer sólidos dispositivos de garantía de la independencia judicial en el plano externo de la magistratura como conjunto y en el plano interno de cada juez como operador individual.

Un segundo ejemplo lo encontramos en la función desempeñada por la corriente denominada del uso alternativo del Derecho o «jurisprudencia alternativa» que tuvo su desarrollo en un contexto socio-jurídico desigualitario, conflictivo y con fuertes residuos de autoritarismo y que, desde ahí, trató de encontrar en la interpretación crítica,

${ }^{20}$ AguILÓ, 2003: «De nuevo sobre "independencia e imparcialidad de los jueces y argumentación jurídica”», Jueces para la democracia, n. ${ }^{\circ} 46$, marzo, p. 48.

${ }_{21}$ AguiLÓ, 2003.

${ }_{22}$ ANDRÉs IbÁÑEZ, Claves, p. 29. PRIETO, 1987: Ideología e interpretación jurídica, Madrid: Tecnos, pp. 42 y ss. MARTín PALlín, 2005: «La formación de los jueces en los procesos de cambio», Derechos, justicia y estado constitucional. Un tributo a Miguel C. Miravet, M. J. AÑón y P. Miravet (eds.), Valencia: Tirant lo Blanch, pp. $155-178$.

${ }^{23}$ ANDRÉs IBÁÑEZ, «Para una ética positiva del juez», art. cit, p. 29. 
proyectiva y emancipatoria de la legalidad constitucional vigente un modelo opuesto al sistema político realmente existente ${ }^{24}$. Este caso tiene interés además porque muestra un doble movimiento por parte de la judicatura. Como escribe Lourdes SouZA ${ }^{25}$ se puede manejar la hipótesis de una línea de continuidad entre el uso alternativo del Derecho y el garantismo, como consecuencia de un giro pragmático originado por el intento de hacer coherente unos presupuestos teórico-prácticos y valorativos que el cambio de los tiempos convertían en desventajosos y nocivos para los propios objetivos, que habría de comportar cambios institucionales de distinto orden en la jurisdicción. Así, cuando la realidad se muestra desigual, injusta y opresora, la lucha por el Derecho pretende su desmantelamiento y supresión. Pero cuando adopta una fisonomía más igualitaria, democrática y justa la estrategia responde a su defensa, mantenimiento y preservación.

Es posible concluir, cautelarmente, que de entre los rasgos institucionales y normativos predicables de la función judicial resulta más distintivo su papel de garante de los derechos que el mantenimiento de la estabilidad del sistema político. Es más, si los jueces contribuyen a articular los puentes de transición en procesos democráticos es fundamentalmente por su condición de garantes de los derechos. Como he descrito, a pesar de las imprecisiones relativas a las notas identificativas del juez en abstracto, entre ellos destacan las exigencias de legitimidad formal procesales o institucionales, especialmente la de ser un tercero supra-partes independiente e imparcial. Desde estos parámetros, tratar en serio a los justiciables como sujeto de derechos puede ser en algunos momentos subversivo.

${ }^{24}$ FerRajoli, 1995: Derecho y Razón. Teoría del galantismo penal, Madrid: Trotta, prólogo de N. BOBBIO. Traducción: P. Andrés Ibáñez, J. C. BAyón, J. Terradillos, A. Ruiz Miguel, R. Cantarero, pp. 835-837 y nota 52.

${ }^{25}$ SouZA, 1998: «Del uso alternativo del derecho al garantismo: una evolución paradójica», Anuario de Filosofía del Derecho, pp. 234, 255. 